•综述・

\title{
榕树种间杂交研究进展
}

\author{
黄建峰 ${ }^{1}$ 徐 点 ${ }^{2}$ 彭艳琼 ${ }^{*}$ \\ 1 (中国科学院西双版纳热带植物园热带森林生态学重点实验室, 云南预腊 666303) \\ 2 (云南省热带作物科学研究所, 云南景洪 666100)
}

\begin{abstract}
摘要: 杂交是生物进化的重要方式和新物种的重要来源, 在植物界普遍存在, 但在不同植物类群中的发生率差异 很大。高度专性传粉体系中, 宿主植物和传粉者经历长期协同进化, 形成远高于其他物种的互利共生机制和合子 前生殖壁垒, 被认为不太可能发生种间杂交。榕树和传粉榕小蜂是动植物间专性传粉关系的典范, 甚至发展出一 对一高度专性关系。但随着研究的深入, 发现了一定程度的宿主转移现象, 引发学者对榕树种间杂交的研究和探 讨。本文从人工杂交、外来种和本地种杂交、岛屿种自然杂交、同域分布近缘种自然杂交, 以及利用系统发育不 一致推测杂交等5个方面, 综述了榕树种间杂交的研究进展, 并对未来研究进行展望。同一亚属内人工榕树种间杂 交 $\mathrm{F}_{1}$ 代一般可育，不同亚属榕树的种间杂交亲和性强度在不同性系统上表现有所不同。种间存在单向或不对称的 双向基因流，依赖专性传粉榕小蜂传粉可能使得渐渗杂交成为榕树种间杂交的主要方式。一系列的合子前隔离机 制, 包括化学隔离、形态隔离、地理隔离、生态隔离、季节隔离等有效维持了榕树物种在遗传和形态上的完整性; 而合子后隔离作用较弱, 传粉榕小蜂在非专性宿主上的繁殖适合度一般表现为显著降低。未来有待在评估杂交对 榕树生物多样性和榕一传粉榕小蜂共生体系稳定性的影响, 分析榕树天然杂交带的杂交模式, 以及探讨影响宿主 转移和榕树杂交的因素等方面开展深入研究。
\end{abstract}

关键词: 榕树; 种间杂交; 宿主转移; 隔离; 传粉榕小蜂

\section{Research progress of interspecific hybridization in genus Ficus}

\author{
Jianfeng Huang ${ }^{1}$, Rui $\mathrm{Xu}^{2}$, Yanqiong Peng ${ }^{1 *}$ \\ 1 CAS Key Laboratory of Tropical Forest Ecology, Xishuangbanna Tropical Botanical Garden, Chinese Academy of \\ Sciences, Mengla, Yunnan 666303 \\ 2 Yunnan Institute of Tropical Crops, Jinghong, Yunnan 666100
}

Abstract: Hybridization plays a vitally important role in biological evolution and speciation. Although
occurring frequently in nature, the prevalence of hybridization events is unevenly distributed across the
plants. It is generally considered unlikely for the obligate insect-pollinated plants, due to the much stronger
prezygotic barriers which were developed during their long co-evolutionary with the host-specific
pollinators, such as the fig-fig-pollinating wasp mutualism. Species-specificity in fig pollination appears to
be extreme compared with most other insect pollination mutualisms. Most fig species are pollinated by only
one wasp species and most wasps are associated with just a single fig species. However, increasing cases of
host shift have been reported with the deepening of research, which raises the research and discussion on
hybridization between host fig species. Here, the research progress on the interspecific hybridization in Ficus
is presented from the following five aspects, artificial hybridization, hybridization between exotic and native
species, natural hybridization of island species, natural hybridization of sympatric allied species, and
hypothesized hybridization by phylogenetic incongruence. Some further research prospects on the Ficus
interbreeding are also outlined. Artificial F $F_{1}$ hybrids between the closely related species of the same subgenus
are usually fertile. However, intensity difference of cross-fertilize compatibility between species of different
subgenera exists between the different breeding systems. There is unidirectional or bidirectional but
asymmetrical interspecific gene flow. Introgression may be the most prominently and prevalently 
hybridization process in Ficus because of the extreme dependence on pollinating fig wasps for sexual reproduction. A series of prezygotic isolation mechanisms maintain the genetic and morphological identity of fig species, including chemical, mechanical, geographic, temporal and ecological isolation. However, the postzygotic isolation is weak effective. The reproductive fitness of pollinating fig wasp is reduced obviously in atypical host species. Future research work needs to be carried out in investigating the effect of hybridization on fig biodiversity and the stability of the fig-fig-pollinating wasp mutualism, and exploring hybridization pattern and consequence of the hybrid zone, and the factors that affect the host shift and hybridization, and so on.

Key words: Ficus; interspecific hybridization; host shift; isolation; pollinating fig wasp

杂交以及随之可能产生的基因渐渗普遍存在 于植物界, 是高等植物基因组进化的重要方式和新 物种的重要来源 (Anderson \& Stebbins, 1954; Ramsey \& Schemske, 2002; Mallet, 2007; Rieseberg \& Willis, 2007)。地球上至少 $25 \%$ 的植物种类涉及到 杂交(Mallet, 2005), 同时, 全球气候变化和人类活 动的日益加剧会降低或者打破物种之间原本形成 的隔离界限, 进一步增加杂交的机会(Vallejo-Marín \& Hiscock, 2016)。然而, 杂交发生率在不同植物类 群中差异很大(Ellstrand et al, 1996), 受生活史、繁 育系统、环境扰动和传粉机制等因素影响 (Grant, 1981), 快速辐射进化的类群杂交发生率非常高 (Mallet, 2007); 而依赖专性传粉者才能完成有性繁 殖的种类被认为不太可能发生杂交(Renoult et al, 2009)。专性传粉体系中, 尤以榕一传粉榕小蜂、丝 兰一丝兰蛾和算盘子一头细蛾三个组合最为经典, 它 们在宿主对传粉者的引诱, 传粉者对宿主的寻找和 识别, 以及双方在物候、形态上高度匹配等一系列 的互利共生机制, 强有力地维持了宿主物种间的合 子前隔离, 导致其杂交难度远高于非专性传粉植物 (Jousselin et al, 2003; Pellmyr, 2003; Moe \& Weiblen, 2012)。然而, 宿主转移打破了宿主一传粉者之间严 格意义上的一对一关系(Menken, 1996; Charleston \& Robertson, 2002; Renoult et al, 2009), 为宿主植物 种间杂交提供了机会。

榕树是榕属(Ficus, Mroaceae)植物的总称, 全 球约 800 余种, 主要分布于热带地区, 是热带植物 区系最大的木本属之一。榕树的生活型极为丰富, 有高大乔木、小乔木、灌木、木质藤本、附生、岩 生、绞杀等几乎所有的木本植物生活型, 占据热带 雨林群落的多个层次, 为众多动物提供食物和栖息 场所, 被公认为是热带雨林中的关键类群(许再富, 1994; Shanahan et al, 2001)。其独特的隐头花序结构
( 以下简称 “榕果”) 需要专一的传粉榕小蜂 (Agaonidae, Hymenoptera)传粉才能完成有性生殖, 而传粉榕小蜂也只能在榕树上繁殖后代, 榕果顶部 的苞片孔是小蜂进入榕果的唯一通道。榕树有雌雄 同株和雌雄异株之分, 各约占榕属种类的一半。其 中雌雄同株榕树同一榕果内既有产生花粉的雄花, 也有产生种子和供小蜂产卵的雌花; 而雌雄异株中 的雌株只有用于产生种子的长柱头雌花, 雄株既有 产生花粉的雄花, 也有供小蜂产卵的短柱头雌花。 宿主榕树和传粉榕小蜂之间互利共生的高度专性 传粉关系甚至被学者用一对一原则(one-to-one rule) 描述(Wiebes, 1963), 即一种榕树只有一种与之对应 的传粉榕小蜂。但随着研究的深入, 发现了越来越 多打破一对一原则的案例(Molbo et al, 2003; Cook \& Segar, 2010; 黄建峰等, 2018), 其中宿主转移导 致一种小蜂为两种或多种榕树传粉的现象使得榕 树种间杂交成为可能, 引发学者对榕树种间杂交的 探讨。本文将从 5 个方面综述榕树种间杂交方面的 研究进展, 并对未来研究进行展望, 以期为榕树及 其他高度专性传粉植物的杂交研究提供参考。

\section{榕树种间杂交研究}

\section{1 人工杂交}

传粉榕小蜂的宿主转移现象很早就有报道 (Müller, 1886; Ramírez, 1970), 引发了学者对榕树 种间杂交的兴趣，但早期针对榕树自然杂交的野外 调查和研究非常少。学者最初通过人工控制实验开 展榕树杂交研究, 相关工作可追溯到为改良品种对 无花果(F. carica, subg. Ficus)不同品种及不同形态 种开展的杂交实验(Hunt, 1911; Condit, 1947)。而不 同物种间的杂交实验始于Condit (1950)将薜荔 (F. pumila, subg. Synoecia)花粉喷酒到无花果雌芯上的 工作，首次获得了可萌发杂交种子, $\mathrm{F}_{1}$ 形态上介于 
双亲之间, 染色体数目与亲本一致 $(2 n=26)$ 。榕树 F. montana分布于东南亚, 小蜂Kradibia tentacularis 为其传粉, 而F. asperifolia分布于非洲热带地区, 两 种榕树系统关系很近, 但地理隔离限制了两者的自 然杂交。Ghana 等 $(2015 a ， b)$ 将携带花粉的 $K$. tentacularis从F. montana雄树引入F. asperifolia䧳树, 得到可育的 $F_{1}$ 代，而后进行 $F_{1}$ 䧳树与 $F$. montana雄 树的回交实验。结果显示: K. tentacularis能在 $\mathrm{F}_{1}$ 和 $F$. asperifolia雄树产卵, 但不能刺激子房形成瘘花, 因 而不能繁殖后代; 但可在回交一代个别雄树的少量 榕果中成功繁殖。表明小蜂的繁殖适合度在 $\mathrm{F}_{1}$ 代中 降低, 而在回交后代中有所提高。另外, 学者还通 过杂交来提高榕树的抗病性。Yakushiji等(2012)利 用无花果作为母本、同属无花果亚属的矮小天仙果 (F. erecta) 作为父本进行人工杂交, 获得了成活率低 $(1.3 \%-2.8 \%)$ 但能够抵抗甘薯长㖨壳菌(Ceratocystis fimbriata)感染的 $\mathrm{F}_{1}$ 代, 可作为砧木来提高无花果抗 溃疡病能力。

上述杂交实验均以雌雄异株榕树为研究对象, 而Ramírez (1986)首次对雌雄同株榕树开展了杂交 实验，将美洲白肉榕亚属(subg. Pharmacosycea)榕 树F. glabrata 与非洲聚果榕亚属(subg. Sycomorus) 的F. sycomorus进行杂交, 虽获得了可萌发杂交种 子, 但 $F_{1}$ 存活期短; 而F. glabrata与同属美洲白肉榕 亚属的F. radula无论正交或反交, 均得到可萌发杂 交种子, 并且 $\mathrm{F}_{1}$ 可育; 但F. glabrata与雌雄异株榕树 F. quercifolia未能产生杂交种子。

上述人工杂交实验和研究表明: 榕树种间能否 杂交, 以及 $\mathrm{F}_{1}$ 代育性与父母本的亲缘关系密切相关, 同亚属内的物种间杂交一般能够成功获得可育的 $\mathrm{F}_{1}$ 代; 不同亚属的物种间存在杂交不亲和性, 但其强 度在不同性系统的榕树上有所不同; 而不同性系统 的榕树之间难以杂交。

\section{2 外来种和本地种杂交}

榕树姿态优美、荫质优良, 常作为绿化观赏树 种被引种到远离其自然分布区的异域, 但往往忽略 了对其传粉者的引种。外来种因缺少专性传粉者, 并克服了与本地种原本存在的地理隔离, 会被本地 种的传粉者拜访, 与本地种杂交, 学者们最初也是 在外来种和本地种之间观察到了榕树的自然杂交 现象。

垂叶榕(F. benjamina)、菩提树 (F. religiosa) 和棱
果榕(F. septica) 原产热带亚洲至大洋洲地区，现已 被广泛栽培。引种到美洲哥斯达黎加的垂叶榕会被 本地种F. padifolia的传粉者Pegoscapus tristani访问, 虽然不能繁殖小蜂后代，但能产生可萌发杂交种子 (Ramírez \& Montero, 1988)。而引种到美国佛罗里达 州的菩提树和棱果榕会被本地种F. aurea的传粉者 Pegoscapus mexicanus传粉，与菩提树杂交产生形 态介于双亲的 $\mathrm{F}_{1}$, 而与棱果榕未产生杂交种子 (Ramírez，1994)。这可能是因为垂叶榕、菩提树、 F. padifolia和F. aurea均为雌雄同株, 且均属于榕亚 属(subg. Urostigma); 而棱果榕为雌雄异株，隶属于 聚果榕亚属, 系统关系较远。然而, Ramírez (1994) 在菲律宾马尼拉发现外来种菩提树与本地种棱果 榕的疑似 $\mathrm{F}_{1}$ 个体, 但因缺少遗传学证据, 很难相信 不同性系统的榕树之间可以杂交。F. lutea (subg. Urostigma, sect. Urostigma)自然分布于热带非洲, 作为观赏树种被引种到南非格雷厄姆斯敦后的 $F$. lutea会被F. thonningii (subg. Urostigma, sect. Galoglychia)和F. sur (subg. Sycomorus, sect. Sycomorus) 的传粉榕小蜂拜访, 并产生可萌发杂交种子, 但 $\mathrm{F}_{1}$ 在子叶期之前全部死亡。F. thonningii的传粉者能在 F. lutea中繁殖, 但F. sur的传粉者却不能(Ware \& Compton, 1992)。这些现象进一步表明种间隔离程 度与亲缘关系密切相关，不同亚属种类之间存在明 显的合子前隔离，而同一亚属的种类未表现出明显 的合子前隔离, 但存在一定程度的合子后隔离; 同 时也为榕树引种与保护提供了借鉴。

\section{3 岛屿种自然杂交}

通过人为打破专一的传粉关系和原本存在的 种间隔离, 例如地理隔离(Ramírez \& Montero, 1988; Ghana et al，2015a，b)以及可能存在的形态隔离 (Condit, 1950)等, 实现人工杂交以及本地种和外来 种的自然杂交，支持自然发生的宿主转移可导致榕 树种间杂交。Janzen (1979)认为宿主转移更容易发 生在岛屿或者条件恶劣的大陆生境(例如分布区的 边缘)。这些生境一方面容易导致传粉者灭绝或暂时 性缺位，为非专性传粉者提供了生态位; 另一方面 也易出现宿主种群过小, 不足以维持传粉者种群的 情况, 促使宿主转移发生。该观点得到了相关研究 的支持(Parrish et al, 2003; Kusumi et al, 2012)。

关于榕树自然杂交以及疑似杂交个体早已有 报道(如: Condit, 1947; Ramírez, 1970), 但首次得到 
遗传学证据支持的案例来自于Parrish等(2003)的研 究。印尼喀拉喀托岛(Krakatau Islands)距最近的大陆 约30 km, 其目前的榕树植物源于1883年火山爆发 后的外来种迁入, 而小蜂种群在榕树迁入很久之后 才逐步建立(Backer，1929；Ernst，1934)。Parrish等 (2003) 在该岛上发现了棱果榕与近缘种水同木 (F. fistulosa) 及对叶榕(F. hispida) 的疑似杂交个体, 暗 示传粉者的暂时性缺位可能促使了宿主转移。他们 从喀拉喀托岛及周边岛屿和邻近大陆采集了包括 棱果榕、水同木和对叶榕在内的7种榕树 64 个个体 以及 22 个疑似杂交个体, 采用 2 个AFLP和 9 个 cpDNA标记, 确认 22 个形态疑似杂交个体中的 6 个 来源于种间杂交, 其中5个来源于棱果榕和水同木 杂交, 1 个来源于棱果榕与对叶榕杂交。这些杂交个 体零散分布于喀拉喀托岛及周边的 Popoli 岛和 Peucang岛, 存在双向基因流, 并检测到回交和基因 渗入证据, 说明 $\mathrm{F}_{1}$ 可育。同样采用AFLP证据, Tsai 等(2015)发现分布于日本Okinoshima岛形态上属于 薜荔的样本中, $28.6 \%$ 的个体来自于薜荔与近缘种 $F$. thunbergii的杂交, 且存在 F. thunbergii向薜荔的单 向基因渗入。薜荔榕果(直径3-4 cm)大于 $F$. thunbergii榕果(直径约 $2 \mathrm{~cm}$ ), 前者的传粉者体型稍大于 后者(Yokoyama \& Iwatsuki, 1998)。榕果大可能暗示 着苞片孔更大, 体型小的小蜂能够较为容易地通过 苞片孔进入大榕果, 相反的情况却很困难; 同时, F. thunbergii在Okinoshima岛的丰富度要低于薜荔, 其 传粉者易遭遇宿主雌花期榕果不足的困境而发生 宿主转移, 这被研究者认为是产生单向基因流的主 要原因(Tsai et al, 2015)。然而, 苞片孔的苞片质地 (例如是否被毛)、数量、排列方式等在种间变异较 大(Verkerke, 1989; 高海燕, $2018^{\circledR}$ ), 并与传粉榕小 蜂的形态特征相互适应(van Noort \& Compton, 1996; Jousselin et al, 2003), 这可能是比差异微小的小蜂 体型更为重要的影响小蜂能否进入榕果的因素, 进 而影响基因流的大小和方向, 相关方面的研究有待 开展。

目前关于榕树杂交产生新物种的研究仅有一 例。起源于火山运动的小笠原群岛 (Ogasawara Islands)距离日本大陆约 $1,000 \mathrm{~km}$, 分布着 3 个榕树

(1) 高海燕 (2018) 基于比较形态解剖学证据的天仙果复合群及其 近缘种的聚类分析. 硕士学位论文, 华东师范大学, 上海.
特有种(F. boninsimae、F. nishimurae和F. iidaiana) 和 1 个外来种(F. microcarpa), 3 个特有种与矮小天仙果 形态相近。Kusumi等(2012)综合 cpDNA和核基因 (ITS、g3pdh、aco1) 证据, 发现3个特有种与日本 Yaeyama群岛分布的矮小天仙果共享cpDNA单倍型, 三者 g3pdh序列完全一致, 并与采自云南的竹叶榕 (F. stenophylla) 以及台湾的蔓榕(F. pedunculosa) 聚 为单系, 与矮小天仙果关系较远。单拷贝基因 $a c 01$ 在F. iidaiana中存在 2 种基因型, 其中 1 种基因型与 另外 2 个特有种及竹叶榕聚为一支, 另外 1 种与矮小 天仙果聚为一支。这种核质基因树的冲突支持 3 个 特有种为杂交起源。而ITS拷贝在特有种间和种内 均有极大变异，且存在假基因，这种不完全一致性 进化的现象可能源于不同亲本拷贝之间相似程度 较低所致; 而假基因化可能源于核仁显性(nucleolar dominance), 即只有遗传自双亲一方的核糖体位点 表现出显性，进一步支持特有种的杂交起源。并且， F. iidaiana为四倍体(Ono, 1990), 通过多倍化实现 了与二倍体榕树的生殖隔离。二倍体榕树 $F$. boninsimae (Ono, 1991) 和F. nishimurae可能起源于 同倍体杂交物种形成，岛屿分布造成的地理隔离和 杂交后代对岛屿极端环境的适应可能在其中发挥 了关键作用(Rieseberg, 1997; Gross, 2012)。然而，同 倍体杂交物种的形成和判定都非常困难, 学者从理 论和实证方面对这种杂交模式进行了探讨。关于 $F$. nishimurae 和 F. boninsimae 是否符合 Schumer 等 (2014)提出的鉴别自然杂交导致物种形成的三个严 格标准, 目前还没有足够证据; 同时, 学者们对该 标准也存在异议(Feliner et al, 2017)。高度专性传粉 植物的同倍体杂交物种形成模式是否表现出新的 特点, 以及大陆分布区的边缘是否也存在广泛的宿 主转移和杂交事件, 有待探讨。

\section{4 同域分布近缘种自然杂交}

宿主转移在榕-传粉榕小蜂体系中广泛存在, 特别是近缘种之间(Cruaud et al, 2012; Yang et al, 2015; 黄建峰等, 2018)。雌花期榕果通过释放化学 挥发物吸引专性传粉者, 是维持专性传粉关系的重 要机制 (Chen et al, 2009; Proffit et al, 2009; Hossaert-McKey et al, 2010)。榕果挥发物是多种有 机化合物的混合, 很多成分被多个物种所共有, 只 是比例不同(Grison-Pigé et al, 2002b), 并且存在种 内变异 (Soler et al, 2011), 还会受到环境影响 
(Grison-Pigé et al, 2002a)。因此不同物种的榕果挥发 物之间并非界限分明，在近缘种间相似度更高 (Cornille et al, 2011; Wang et al, 2016), 导致宿主转 移更易发生于近缘种之间, 其发生率深刻影响着种 间基因流的大小和榕树物种的独立性程度。对同域 分布的近缘种开展宿主转移和杂交研究, 能够更好 地理解榕树物种分化和隔离机制的进化。

Ficus bernaysii、F. congesta、F. hahliana、F. hispidioides、F. morobensis和F. pachyrrhachis为聚果榕 亚属对叶榕组(sect. Sycocarpus)的6种雌雄异株榕树, 研究发现同域分布于巴布亚新几内亚低地雨林的 这6种榕树存在极低的宿主转移率(0-3.2\%) (Moe et al, 2011), 微卫星数据检测到 $1 \%-2 \%$ 的样本涉及杂 交(Moe \& Weiblen, 2012)。以F. hispidioides为母本, 分别以F. congesta、F. hahliana、F. morobensis、F. pachyrrhachis 为父本进行交叉放蜂实验, 除 $F$. hahliana $\times F$. hispidioides组合之外, 其他组合均获 得了可萌发杂交种子。将 4 种父本的传粉者引入 $F$. hispidioides雄树榕果后均能够产卵形成㾉花, 但成 功率显著降低, 这可能源于小蜂产卵器长度与非专 性宿主花柱长度不匹配导致的形态隔离(Weiblen, 2004); 但小蜂后代均不能发育到成虫期, 暗示还存 在生理上的隔离。同时, 研究者还发现小蜂在进入 榕果之前有选择和识别宿主的能力, 在进入榕果之 后即丧失宿主识别能力, 不管是否为其专性宿主, 均会进行传粉和产卵, 这可能是小蜂触角在通过苞 片孔的过程中受到损坏的缘故(Moe \& Weiblen, 2012)。

木瓜榕复合体 (subg. Sycomorus, sect. Sycomorus) 是国内学者关注较多的类群, 主要包括木瓜 榕(F. auriculata)、苹果榕(F. oligodon)和海南榕(F. hainanensis)这3个分布区重叠的雌雄异株类群。三 者在分类学上的争议由来已久, 一般将其处理为木 瓜榕的3个形态种, 而最新的研究建议将三者归入 同一物种(Zhang et al, 2018)。三者之间存在一定程 度的宿主转移(Berg, 2007; Kuaraksa et al, 2012; Wei et al, 2014), 并发现疑似杂交个体(Corner, 1978)。交 叉放蜂实验表明两两杂交组合均可产生 $F_{1}$ 代, 传粉 榕小蜂也可在非专性宿主中繁殖(杨培, 2010; 魏作 东, 2010), 但海南榕传粉者在木瓜榕上的适合度降 低(Yang et al, 2012)。微卫星数据显示6\%的木瓜榕 复合体样本涉及到杂交和渗入, 其中木瓜榕和苹果
榕的杂交后代占4.5\% (魏作东，2010; Wei et al, 2014)。同时，在木瓜榕复合体和同域分布的鸡嗉子 榕(F. semicordata, subg. Sycomorus, sect. hemicardia) 的两个变种, 即大果鸡嗉子榕 (F. semicordata var. semicordata)和小果鸡嗉子榕 (F. semicordata var. montana)之间也检测到基因流; 两个变种之间也可 杂交产生 $F_{1}$ 代(Wang et al, 2013)。对木瓜榕复合体和 鸡嗉子榕两变种 5 个类群的综合研究表明：榕果挥 发物的相似度、宿主转移频率和种间基因交流水平 三者之间存在明显的正相关(Wang et al, 2016)。

上述研究表明, 除了榕果挥发物导致的化学隔 离之外, 生境偏好产生的生态隔离和物候分化产生 的季节隔离(Wei et al, 2014), 以及小蜂产卵器和宿 主花柱长度不匹配产生的形态隔离和导致小蜂繁 殖适合度降低的生理隔离(Moe \& Weiblen, 2012)等, 都对维持同域分布近缘种的物种独立性发挥着重 要作用。

\section{5 利用系统发育不一致推测杂交}

利用不同类型基因的系统发育不一致(phylogenetic incongruence)判断种间杂交是比较普遍的 方法，也是首选的鉴别手段(Aronld，1997; 王玉国， 2017)。榕树研究中主要利用核基因和cpDNA基因 之间系统关系的不一致来推测杂交。另外，榕树和 传粉榕小蜂高度专性的传粉关系以及长期的协同 成种过程(Cruaud et al, 2012), 导致两者在系统发育 上有很好的一致性; 相反地, 可通过两类群在系统 发育关系上的不一致来推测宿主转移事件，进而推 测种间杂交，这也是推测榕树杂交的重要方法之一 (Bruun-Lund et al, 2017)。

Machado等(2005)综合前人研究发现，榕树和 传粉榕小蜂在系统发育上存在大量由宿主转移导 致的不一致现象，并结合种间杂交案例提出：杂交 和渐渗可能是榕树物种多样性形成的主要机制。系 统发育研究发现榕亚属sect. Galoglychia类群与其 传粉榕小蜂在系统发育上存在不一致(Erasmus et al, 2007; Rønsted et al, 2007), 暗示存在宿主转移。 Renoult等(2009)进一步利用cpDNA和核基因证据, 发现sect. Galoglychia类群存在多处明显的核质系 统树冲突，但发生率在不同亚组中明显不同，并认 为这种冲突源于基因渗入。存在核质系统树冲突的 物种，与其传粉者在系统发育上也存在不一致，支 持宿主转移导致了种间杂交和渐渗。通过对榕果直 
径、小蜂体长、宿主转移和核质系统树冲突的相关 性分析, 发现榕果小(对应的小蜂体型一般较小)和 小蜂体型小的类群更易发生宿主转移(Renoult et al, 2009)。另外，这种核质系统树的不一致，以及榕树 和传粉榕小蜂系统发育关系的不一致, 在榕树系统 的深层分支(deep clade)和浅层分支(shallow clade) 上都存在(Cruaud et al, 2012; Bruun-Lund et al, 2017), 暗示宿主转移和种间杂交可能贯穿于榕-传 粉榕小蜂共生体系的整个发展进程中。

综合核质系统树以及榕树和传粉榕小蜂系统 树之间的冲突，对大范围检测榕树杂交、推断宿主 转移和杂交事件具有重要价值。但传粉者的重复成 种, 而后出现宿主和传粉者协同成种, 以及不对称 灭绝，同样会导致宿主和传粉者系统发育不一致， 效果类似于宿主转移(Reed et al, 2007); 同时, 核质 系统树不一致在植物类群中非常普遍，除了杂交之 外, 基因水平转移、基因复制后的拷贝丢失、不完 全谱系分选、趋同进化、长支吸引等均可能导致核 质系统树冲突(Wendel \& Doyle, 1998; Kennedy et al, 2005), 须引起注意。

\section{研究展望}

目前关于榕树杂交方面的研究还很少, 随着高 通量测序和基因组技术的飞速发展, 越来越多新技 术用于物种杂交研究中, 基于系统发育和群体遗传 的分析手段也逐渐发展为系统发育基因组学和种 群基因组学(毛建丰等, 2017; 王玉国，2017)。例如 RAD-seq测序、全基因组测序、转录组测序、单核 苷酸多态性微阵列(SNP array)技术等，已用于不同 动植物类群的杂交研究中(Renaut et al, 2013; Burri et al, 2015; Liu KJ et al, 2015; Rutledge et al, 2015), 这也为榕树种间杂交研究提供了新的契机。基于 RAD-seq技术开展的榕树系统发育基因组学研究在 探讨榕树杂交方面有良好表现(Bruun-Lund et al, 2017), 并对目前该类群进化方面的一些主流观点 提出了挑战(Rasplus et al, 2018; Wang et al, 未发表 数据), 显示出巨大的应用潜力。未来可在以下几个 方面对榕树杂交开展深入研究, 以期更好地认识榕 一传粉榕小蜂体系的协同进化历史, 对其他专性传 粉体系也有重要参考价值。

\section{1 评估杂交对榕树生物多样性的影响}

目前对榕属物种多样性的形成主要有两种观
点。协同系统发育 (cophylogeny)研究认为, 与传粉 榕小蜂的协同成种是榕树物种多样性产生的主要 机制(Cruaud et al, 2012), 这也是目前比较主流的观 点，得到很多研究的支持(Herre et al, 1996; Rønsted et al, 2005; Cruaud et al, 2011)。而Machado等(2005) 认为杂交和渐渗丰富了榕树的物种多样性, 但相关 研究较少, 也很少有研究关注种间杂交对榕树生物 多样性的影响, 有待未来研究评估杂交在榕树物种 多样性形成中所扮演的角色。

\section{2 天然杂交带的研究有待开展和深入}

作为研究种间杂交的天然实验室，杂交带 (hybrid zone)已成为植物系统与进化领域的研究热 点之一, 为探讨物种形成和适应机制、种间隔离和 遗传分化维持机制等提供了绝佳机遇(Jiggins \& Mallet, 2000; Abbott et al, 2013; Abbott, 2017)。榕树 种类丰富，种间存在广泛的同域分布现象。在综合 形态、化学、物候和遗传学等多方面证据的基础上, 开展榕树天然杂交带同域分布近缘种间的基因流 动态、物种完整性和杂合子适应性进化研究，对理 解榕树生物多样性的产生机制、宿主转移的发生机 制、种间隔离机制等都可能发挥关键作用，但目前 该方面的研究还是空白。岛屿和边缘分布区的生境 压力更大，更容易出现传粉榕小蜂的宿主转移，因 此也最有可能出现杂交带。已报道了多例岛屿分布 榕树物种的种间自然杂交现象，为杂交带的研究提 供了很好的前期基础。与相对单一的岛屿生境相比, 大陆分布区生境梯度变化更为明显，边缘分布区生 境更为复杂，更适于开展榕树的种群分化、局域适 应(local adaptation)、种间隔离的形成、杂交带的稳 定以及随全球气候变化的动态移动(Abbott, 2017) 等方面的研究, 有必要在未来研究中给予更多关注, 借助种群基因组学分析能够清楚地认识杂交带的 遗传结构及形成过程(Hemmer-Hansen et al, 2014; Nadeau et al, 2014)。

\section{3 影响榕树杂交的因素有待深入研究}

研究表明雌雄异株比雌雄同株榕树的宿主专 一性更强, 宿主转移在雌雄同株榕树中的发生率更 高(Moe et al, 2011; Yang et al, 2015); 同时，相对于 雌雄异株榕树传粉者较短的飞行距离(Wang et al, 2009; Chen et al, 2012), 雌雄同株榕树传粉者的飞 行距离要远得多(Nason et al, 1998; Ahmed et al, 2009), 会削弱地理隔离的作用，因此其合子前隔离 
作用会更弱。同一亚属内的雌雄同株榕树之间 (Ramírez, 1986, 1994; Ramírez \& Montero, 1988)也 未表现出明显的合子后隔离, 据此推断近缘的雌雄 同株榕树会有更高的杂交发生率; 然而, 个例研究 表明不同亚属的雌雄同株物种间存在远缘杂交不 亲和性(Ramírez, 1986; Ware \& Compton, 1992), 而 不同亚属的雌雄异株榕树之间能够杂交(Condit, 1950), 暗示不同性系统榕树的合子后隔离强度不 同，雌雄异株类型的合子后隔离作用可能更强。但 目前榕树杂交方面的研究主要集中在雌雄异株种 类。虽有一例不同性系统榕树之间疑似杂交个体的 报道(Ramírez \& Montero, 1988; Ramírez, 1994), 但 无遗传学证据, 我们对此表示怀疑。不同性系统榕 树的杂交发生率和隔离强度是否明显不同, 以及不 同性系统榕树之间能否杂交等问题有待更多研究。

另外, 榕果和小蜂体型大小导致宿主转移和种 间杂交的发生率在榕树不同亚组中并不均衡, 种间 双向基因流也并不对称(Renoult et al, 2009; Moe et al, 2011; Tsai et al, 2015)。然而这种情况在木瓜榕复 合体和鸡嗉子榕上似乎并不适用(Wang et al, 2016), 表明不同类群中影响宿主转移和杂交的因素不同, 可能还受到其他诸如苍片孔的苍片质地、数量、排 列方式等多方面因素的影响, 有待综合形态、化学 挥发物以及遗传学等证据, 在榕属多个亚属开展更 广泛的比较分析。

\section{4 杂交对榕一传粉榕小蜂共生体系稳定性的影响}

榕树和小蜂繁殖都依赖榕果内雌花, 导致对雌 花资源的争夺, 在雌雄同株榕树上表现得更为突出, 宿主惩罚效应(host sanction)被认为是榕树权衡雌花 资源分配, 维持榕-传粉榕小蜂共生体系长期稳定 的关键机制, 其丧失或减弱会导致主动传粉类型的 榕树出现只产卵不传粉的欺骗者小蜂, 但被动传粉 榕树无宿主惩罚效应(Jandér \& Herre, 2010; Jandér et al, 2012)。Herre等(2008)推测主动传粉榕树宿主 惩罚效应的丧失或减弱可能源于其与被动传粉榕 树之间的杂交和基因渗入。目前只在F. sycomorus (Compton et al, 1991)、高山榕(F. altissima) (Peng et al, 2008)和榕树 (F. microcarpa) (Martinson et al, 2014)中发现了欺骗者, 研究也表明主动传粉类型 的高山榕丧失了宿主惩罚效应(赵江波, 2013), 但还 没有深层的原因解释。榕树杂交研究有助于揭示宿
主惩罚效应的丧失和传粉者欺骗性的进化机制。

\section{3 结语}

榕树和传粉榕小蜂是动植物间专性传粉关系 的典范，但也非严格的一对一关系，存在许多古老 和近期的宿主转移及种间杂交事件。多倍化、同倍 体杂交物种形成和渐渗杂交三种主要的杂交方式 (Rieseberg, 1997; Mallet, 2007; 王玉国, 2017)可能 在榕树中都存在。而特殊的隐头花序和䧳雄异熟机 制使得 $\mathrm{F}_{1}$ 无法自交，必然接受亲本传粉者传粉产生 回交，导致渐渗杂交可能是最主要的方式。这使得 榕树并非简单的二歧分化，而是呈现出复杂的网状 进化; 与传粉榕小蜂也并非严格的协同进化，而是 兼具严格和发散的协同进化关系(Janzen，1980; Machado et al, 2005; 陈艳等, 2010)。由于单个榕果 就可产生成百上千头小蜂, 极低的宿主转移发生率 也会产生大量的杂交事件，但事实上即使同域分布 的近缘种也能很好地维持其形态和遗传上的独立

性 (Moe \& Weiblen, 2012; Wei et al, 2014; Souto-Vilarós et al, 2018)。除了最主要的化学和形态 隔离之外, 地理隔离(Kusumi et al, 2012; Ghana et al, 2015a, b)、生态隔离和季节隔离(Wei et al, 2014; Liu GX et al, 2015), 以及榕果表面的物理和化学特征 (Ware \& Compton, 1992; Gibernau et al, 1998; Wang et al, 2013)等也发挥着重要的合子前隔离作用。合 子后隔离主要体现在不同亚属的物种间, 例如杂种 不活(Ramírez, 1986; Ware \& Compton, 1992)。小蜂 在非专性宿主上适合度一般表现为显著降低，包括 不能形成瘞花(Gahana et al, 2015a, b)、产卵成功率 降低和后代不能发育成熟(Moe \& Weiblen, 2012)等, 都会促使小蜂寻找专性宿主，降低杂交发生率，对 维持榕-传粉榕小蜂共生体系的传粉专一性具有重 要意义。

杂交在生物进化过程中的重要性已有大量阐 述(Rieseberg, 1997; Mallet et al, 2007; Abbott et al, 2013), 日益加剧的全球气候变化、人类活动干扰和 生物入侵导致的杂交问题更加突出，越来越受到关 注(Vilà et al, 2000; Vallejo-Marín \& Hiscock, 2016)。 宿主-传粉者高度专性传粉体系中, 由于传粉昆虫 的单一性，宿主的有性繁殖过程更容易受环境扰动 和人类活动的影响, 进而增加宿主转移和宿主植物 
杂交的机会，但相关类群杂交领域的研究相对较 少。目前榕-传粉榕小蜂研究领域的多数学者对杂 交在该共生体系中扮演的角色或重要性持怀疑态 度, 借助新技术开展深入的种间杂交研究对探讨榕 树丰富物种多样性和生活型的产生、生态适应性、 传粉欺骗者的出现和榕蜂共生体系的稳定、种间隔 离机制的进化、榕树引种与保护等都具有重要价值, 对其他专性传粉植物的杂交研究也具有借鉴意义。

\section{参考文献}

Abbott R, Albach D, Ansell S, Arntzen JW, Baird SJE, Bierne N, Boughman J, Brelsford A, Buerkle CA, Buggs R, Butlin RK, Dieckmann U, Eroukhmanoff F, Grill A, Cahan SH, Hermansen JS, Hewitt G, Hudson AG, Jiggins C, Jones J, Keller B, Marczewski T, Mallet J, Martinez-Rodriguez P, Möst M, Mullen S, Nichols R, Nolte AW, Parisod C, Pfennig K, Rice AM, Ritchie MG, Seifert B, Smadja CM, Stelkens R, Szymura JM, Väinölä R, Wolf JBW, Zinner D (2013) Hybridization and speciation. Journal of Evolutionary Biology, 26, 229-246.

Abbott RJ (2017) Plant speciation across environmental gradients and the occurrence and nature of hybrid zones. Journal of Systematics and Evolution, 55, 238-258.

Ahmed S, Compton SG, Butlin RK, Gilmartin PM (2009) Wind-borne insects mediate directional pollen transfer between desert fig trees 160 kilometers apart. Proceedings of the National Academy of Sciences, USA, 106, 2034220347.

Anderson E, Stebbins GL (1954) Hybridization as an evolutionary stimulus. Evolution, 8, 378-388.

Arnold ML (1997) Natural Hybridization and Evolution. Oxford University Press, Oxford.

Backer CA (1929) The Problem of Krakatoa as Seen by a Botanist. Published by the author, Sourabaya.

Berg CC (2007) Precursory taxonomic studies on Ficus (Moraceae) for the Flora of Thailand. Thai Forest Bulletin, 35, 4-28.

Bruun-Lund S, Clement WL, Kjellberg F, Rønsted N (2017) First plastid phylogenomic study reveals potential cyto-nuclear discordance in the evolutionary history of Ficus L. (Moraceae). Molecular Phylogenetics and Evolution, 109, 93-104.

Burri R, Nater A, Kawakami T, Mugal CF, Olason PI, Smeds L, Suh A, Dutoit L, Bureš S, Garamszegi LZ, Hogner S, Moreno J, Qvarnström A, Ružić M, Sæther SA, Sætre GP, Török J, Ellegren H (2015) Linked selection and recombination rate variation drive the evolution of the genomic landscape of differentiation across the speciation continuum of Ficedula flycatchers. Genome Research, 25, 1656-1665.

Charleston MA, Robertson DL (2002) Preferential host switching by primate lentiviruses can account for phylogenetic similarity with the primate phylogeny. Systematic Biology, 51, 528-535.

Chen C, Song Q, Proffit $\mathrm{M}$, Bessière JM, Li Z, Hossaert-McKey M (2009) Private channel: A single unusual compound assures specific pollinator attraction in Ficus semicordata. Functional Ecology, 23, 941-950.

Chen Y, Compton SG, Liu M, Chen XY (2012) Fig trees at the northern limit of their range: The distributions of cryptic pollinators indicate multiple glacial refugia. Molecular Ecology, 21, 1687-1701.

Chen Y, Li HQ, Liu M, Chen XY (2010) Species-specificity and coevolution of figs and their pollinating wasps. Biodiversity Science, 18, 1-10. (in Chinese with English abstract) [陈艳，李宏庆，刘敏，陈小勇 (2010) 榕一传粉 榕小蜂间的专一性与协同进化. 生物多样性, 18, 1-10.]

Compton SG, Holton KC, Rashbrook VK, van Noort S, Vincent SL (1991) Studies of Ceratosolen galili, a non-pollinating agaonid fig wasp. Biotropica, 23, 188-194.

Condit IJ (1947) The Fig. Chronica Botanica Co., Waltham.

Condit IJ (1950) An interspecific hybrid in Ficus. Journal of Heredity, 41, 165-168.

Cook JM, Segar ST (2010) Speciation in fig wasps. Ecological Entomology, 35, 54-66.

Corner EJH (1978) Ficus dammaropsis and the multibracteate species of Ficus sect. Sycocarpus. Philosophical Transactions of the Royal Society B: Biological Sciences, 281, 373-406.

Cornille A, Underhill JG, Cruaud A, Hossaert-McKey M, Johnson SD, Tolley KA, Kjellberg F, van Noort S (2011) Floral volatiles, pollinator sharing and diversification in the fig-wasp mutualism: Insights from Ficus natalensis, and its two wasp pollinators (South Africa). Proceedings of the Royal Society of London B: Biological Sciences, 279, 1731-1739.

Cruaud A, Cook J, Yang DR, Genson G, Jabbour-Zahab R, Kjellberg F, Pereira RAS, Rønsted N, Santos O, Savolainen V, Ubaidillah R, van Noort S, Peng YQ, Rasplus JY (2011) Fig-fig wasp mutualism: The fall of the strict cospeciation paradigm? In: Evolution of Plant-Pollinator Relationships (ed. Patiny S), pp. 68-102. Cambridge University Press, Cambridge.

Cruaud A, Rønsted N, Chantarasuwan B, Chou LS, Clement WL, Couloux A, Cousins B, Genson G, Harrison RD, Hanson PE, Hossaert-Mckey M, Jabbour-Zahab R, Jousselin E, Kerdelhué C, Kjellberg F, Lopez-Vaamonde C, Peebles J, Peng YQ, Pereira RAS, Schramm T, Ubaidillah R, van Noort S, Weiblen GD, Yang DR, Yodpinyanee A, Libeskind-Hadas R, Cook JM, Rasplus JY, Savolainen V (2012) An extreme case of plant-insect co-diversification: Figs and fig-pollinating wasps. Systematic Biology, 61, 1029-1047.

Ellstrand NC, Whitkus R, Rieseberg LH (1996) Distribution of spontaneous plant hybrids. Proceedings of the National Academy of Sciences, USA, 93, 5090-5093. 
Erasmus JC, van Noort S, Jousselin E, Greeff JM (2007) Molecular phylogeny of fig wasp pollinators (Agaonidae, Hymenoptera) of Ficus section Galoglychia. Zoologica Scripta, 36, 61-78.

Ernst A (1934) Das biologische Krakatauproblem. Vierteljahrsschrift der Naturforschenden Gesellschaft in Zuerich, 4, 240-244.

Feliner GN, Álvarez I, Fuertes-Aguilar J, Heuertz M, Marques I, Moharrek F, Piñeiro R, Riina R, Rosselló JA, Soltis PS, Villa-Machío I (2017) Is homoploid hybrid speciation that rare? An empiricist’s view. Heredity, 118, 513-516.

Ghana S, Suleman N, Compton SG (2015a) A comparison of pollinator fig wasp development in figs of Ficus montana and its hybrids with Ficus asperifolia. Entomologia Experimentalis et Applicata, 156, 225-237.

Ghana S, Suleman N, Compton SG (2015b) Ability to gall: The ultimate basis of host specificity in fig wasps? Ecological Entomology, 40, 280-291.

Gibernau M, Hossaert-McKey M, Frey J, Kjellberg F (1998) Are olfactory signals sufficient to attract fig pollinators? Ecoscience, 5, 306-311.

Grant V (1981) Plant speciation. Science, 317, 910-914.

Grison-Pigé L, Bessière JM, Hossaert-McKey M (2002a) Specific attraction of fig-pollinating wasps: Role of volatile compounds released by tropical figs. Journal of Chemical Ecology, 28, 283-295.

Grison-Pigé L, Hossaert-McKey M, Greeff JM, Bessière JM (2002b) Fig volatile compounds-A first comparative study. Phytochemistry, 61, 61-71.

Gross BL (2012) Genetic and phenotypic divergence of homoploid hybrid species from parental species. Heredity, 108, 157-158.

Hemmer-Hansen J, Therkildsen NO, Pujolar JM (2014) Population genomics of marine fishes: Next-generation prospects and challenges. The Biological Bulletin, 227, 117-132.

Herre EA, Jandér KC, Machado CA (2008) Evolutionary ecology of figs and their associates: Recent progress and outstanding puzzles. Annual Review of Ecology, Evolution, and Systematics, 39, 439-458.

Herre EA, Machado CA, Bermingham E, Nason JD, Windsor DM, McCafferty S, Van Houten W, Bachmann K (1996) Molecular phylogenies of figs and their pollinator wasps. Journal of Biogeography, 23, 521-530.

Hossaert-McKey M, Soler C, Schatz B, Proffit M (2010) Floral scents: Their roles in nursery pollination mutualisms. Chemoecology, 20, 75-88.

Huang JF, Xu R, Peng YQ (2018) Progress on the breakdown of one-to-one rule in symbiosis of figs and their pollinating wasps. Biodiversity Science, 26, 295-303. (in Chinese with English abstract) [黄建峰, 徐睿, 彭艳琼 (2018) 榕-传粉 榕小蜂非一对一共生关系的研究进展. 生物多样性, 26, 295-303.]

Hunt BW (1911) Fig breeding. Bulletin of the University of Georgia, 11, 146-148.
Jandér KC, Herre EA (2010) Host sanctions and pollinator cheating in the fig tree-fig wasp mutualism. Proceedings of the Royal Society of London B: Biological Sciences, 277, 1481-1488.

Jandér KC, Herre EA, Simms EL (2012) Precision of host sanctions in the fig tree-fig wasp mutualism: Consequences for uncooperative symbionts. Ecology Letters, 15, 13621369.

Janzen DH (1979) How to be a fig. Annual Review of Ecology and Systematics, 10, 13-51.

Janzen DH (1980) When is it coevolution. Evolution, 34, 611-612.

Jiggins CD, Mallet J (2000) Bimodal hybrid zones and speciation. Trends in Ecology and Evolution, 15, 250-255.

Jousselin E, Rasplus JY, Kjellberg F (2003) Convergence and coevolution in a mutualism: Evidence from a molecular phylogeny of Ficus. Evolution, 57, 1255-1269.

Kennedy M, Holland BR, Gray RD, Spencer HG (2005) Untangling long branches: Identifying conflicting phylogenetic signals using spectral analysis, neighbor-net, and consensus networks. Systematic Biology, 54, 620-633.

Kuaraksa C, Elliott S, Hossaert-Mckey M (2012) The phenology of dioecious Ficus spp. tree species and its importance for forest restoration projects. Forest Ecology and Management, 265, 82-93.

Kusumi J, Azuma H, Tzeng HY, Chou LS, Peng YQ, Nakamura K, Su ZH (2012) Phylogenetic analyses suggest a hybrid origin of the figs (Moraceae: Ficus) that are endemic to the Ogasawara (Bonin) Islands, Japan. Molecular Phylogenetics and Evolution, 63, 168-179.

Liu GX, Yang DR, Peng YQ, Compton SG (2015) Complementary fruiting phenologies facilitate sharing of one pollinator fig wasp by two fig trees. Journal of Plant Ecology, 8, 197-206.

Liu KJ, Steinberg E, Yozzo A, Song Y, Kohn MH, Nakhleh L (2015) Interspecific introgressive origin of genomic diversity in the house mouse. Proceedings of the National Academy of Sciences, USA, 112, 196-201.

Machado CA, Robbins N, Gilbert MTP, Herre EA (2005) Critical review of host specificity and its coevolutionary implications in the fig/fig-wasp mutualism. Proceedings of the National Academy of Sciences, USA, 102, 6558-6565.

Mallet J (2005) Hybridization as an invasion of the genome. Trends in Ecology and Evolution, 20, 229-237.

Mallet J (2007) Hybrid speciation. Nature Reviews, 446, 279283.

Mao JF, Ma YP, Zhou RC (2017) Approaches used to detect and test hybridization: Combining phylogenetic and population genetic analyses. Biodiversity Science, 25, 577-599. (in Chinese with English abstract) [毛建丰, 马永鹏, 周仁 超 (2017) 结合系统发育与群体遗传学分析检验杂交是 否存在的技术策略. 生物多样性, 25, 577-599.]

Martinson EO, Jandér KC, Peng YQ, Chen HH, Machado CA, Arnold AE, Herre EA (2014) Relative investment in egg 
load and poison sac in fig wasps: Implications for physiological mechanisms underlying seed and wasp production in figs. Acta Oecologica, 57, 58-66.

Menken SB (1996) Pattern and process in the evolution of insect-plant associations: Yponomeuta as an example. Entomologia Experimentalis et Applicata, 80, 297-305.

Moe AM, Rossi DR, Weiblen GD (2011) Pollinator sharing in dioecious figs (Ficus: Moraceae). Biological Journal of the Linnean Society, 103, 546-558.

Moe AM, Weiblen GD (2012) Pollinator-mediated reproductive isolation among diocious fig species (Ficus, Moraceae). Evolution, 66, 3710-3721.

Molbo D, Machado CA, Sevenster JG, Keller L, Herre EA (2003) Cryptic species of fig-pollinating wasps: Implications for the evolution of the fig-wasp mutualism, sex allocation, and precision of adaptation. Proceedings of the National Academy of Sciences, USA, 100, 5867-5872.

Müller F (1886) Feigenwespen. Kosmos, 18, 55-62.

Nadeau NJ, Ruiz M, Salazar P, Counterman B, Medina JA, Ortiz-Zuazaga H, Morrison A, McMillan WO, Jiggins CD, Papa R (2014) Population genomics of parallel hybrid zones in the mimetic butterflies, $H$. melpomene and $H$. erato. Genome Research, 24, 1316-1333.

Nason JD, Herre EA, Hamrick JL (1998) The breeding structure of a tropical keystone plant resource. Nature, 391, 685-687.

Ono M (1990) Chromosome number of some endemic plant species of the Bonin Islands III. Ogasawara Research, 16, $1-27$.

Ono M (1991) The flora of the Bonin (Ogasawara) Islands. Aliso: A Journal of Systematic and Evolutionary Botany, 13, 95-105.

Parrish TL, Koelewijn HP, van Dijk PJ, Kruijt M (2003) Genetic evidence for natural hybridization between species of dioecious Ficus on island populations. Biotropica, 35, 333-343.

Peng YQ, Duan ZB, Yang DR, Rasplus JY (2008) Co-occurrence of two Eupristina species on Ficus altissima in Xishuangbanna, SW China. Symbiosis, 45, 9-14.

Pellmyr O (2003) Yuccas, yucca moths, and coevolution: A review. Annals of Missouri Botanical Garden, 90, 35-55.

Proffit M, Chen C, Soler C, Bessiere JM, Schatz B, Hossaert-McKey M (2009) Can chemical signals, responsible for mutualistic partner encounter, promote the specific exploitation of nursery pollination mutualisms? The case of figs and fig wasps. Entomologia Experimentalis et Applicata, 131, 46-57.

Ramírez BW (1970) Taxonomic and biological studies of neotropical fig wasps (Hymenoptera: Agaonidae). The University of Kansas Science Bulletin, 49, 1-44.

Ramírez BW (1986) Artificial hybridization and self-fertilization in Ficus (Moraceae). Brenesia, 25, 265-272.

Ramírez BW (1994) Hybridization of Ficus religiosa with F. septica and F. aurea (Moraceae). Revista de Biología
Tropical, 42, 339-342.

Ramírez BW, Montero SJ (1988) Ficus microcarpa L., F. benjamina L. and other species introduced in the New World, their pollinators (Agaonidae) and other fig wasps. Revista de Biología Tropical, 36, 441-446.

Ramsey J, Schemske DW (2002) Neopolyploidy in flowering plants. Annual Review of Ecology and Systematic, 33, 589-639.

Rasplus JY, Rodriguez LJ, Tollon-Cordet C, Cruaud A (2018) Revisiting the phylogeny of Ficus (Moraceae): When next generation sequencing corroborates past generation botanists. BioRxiv, 340463.

Reed DL, Light JE, Allen JM, Kirchman JJ (2007) Pair of lice lost or parasites regained: The evolutionary history of anthropoid primate lice. BMC Biology, 5, 7-17.

Renaut S, Grassa CJ, Yeaman S, Moyers BT, Lai Z, Kane NC, Bowers JE, Burke JM, Rieseberg LH (2013) Genomic islands of divergence are not affected by geography of speciation in sunflowers. Nature Communications, 4, 1827.

Renoult JP, Kjellberg F, Grout C, Santoni S, Khadari B (2009) Cyto-nuclear discordance in the phylogeny of Ficus section Galoglychia and host shifts in plant-pollinator associations. BMC Evolutionary Biology, 9, 248-265.

Rieseberg LH (1997) Hybrid origins of plant species. Annual Review of Ecology and Systematics, 28, 359-389.

Rieseberg LH, Willis JH (2007) Plant speciation. Science, 317, 910-914.

Rønsted N, Salvo G, Savolainen V (2007) Biogeographical and phylogenetic origins of African fig species (Ficus section Galoglychia). Molecular Phylogenetics and Evolution, 43, 190-201.

Rønsted N, Weiblen GD, Cook JM, Salamin N, Machado CA, Savolainen P (2005) 60 million years of co-divergence in the fig-wasp symbiosis. Proceedings of the Royal Society of London B: Biological Sciences, 272, 2593-2599.

Rutledge LY, Devillard S, Boone JQ, Hohenlohe PA, White BN (2015) RAD sequencing and genomic simulations resolve hybrid origins within North American Canis. Biology Letters, 11, 20150303.

Schumer M, Rosenthal GG, Andolfatto P (2014) How common is homoploid hybrid speciation? Evolution, 68, 1553-1560.

Shanahan M, So S, Compton SG, Corlett R (2001) Fig-eating by vertebrate frugivores: A global review. Biological Reviews, 76, 529-572.

Soler C, Hossaert-McKey M, Buatois B, Bessière JM, Schatz B, Proffit M (2011) Geographic variation of floral scent in a highly specialized pollination mutualism. Phytochemistry, 72, 74-81.

Souto-Vilarós D, Proffit M, Buatois B, Rindos M, Sisol M, Kuyaiva T, Isua B, Michalek J, Darwell CT, HossaertMcKey M, Weiblen GD, Novotny V, Segar ST (2018) Pollination along an elevational gradient mediated both by floral scent and pollinator compatibility in the fig and fig-wasp mutualism. Journal of Ecology, 106, 2256-2273. 
Tsai L, Hayakawa H, Fukuda T, Yokoyama J (2015) A breakdown of obligate mutualism on a small island: An interspecific hybridization between closely related fig species (Ficus pumila and Ficus thunbergii) in Western Japan. American Journal of Plant Sciences, 6, 126-131.

Vallejo-Marín M, Hiscock SJ (2016) Hybridization and hybrid speciation under global change. New Phytologist, 211, 1170-1187.

van Noort S, Compton SG (1996) Convergent evolution of agaonine and sycoecine (Agaonidae, Chalcidoidea) head shape in response to the constraints of host fig morphology. Journal of Biogeography, 23, 415-424.

Verkerke W (1989) Structure and function of the fig. Experientia, 45, 612-622.

Vilà M, Webe, E, Antonio CM (2000) Conservation implications of invasion by plant hybridization. Biological Invasions, 2, 207-217.

Wang G, Cannon CH, Chen J (2016) Pollinator sharing and gene flow among closely related sympatric dioecious fig taxa. Proceedings of the Royal Society B: Biological Sciences, 283, 20152963.

Wang G, Compton SG, Chen J (2013) The mechanism of pollinator specificity between two sympatric fig varieties: A combination of olfactory signals and contact cues. Annals of Botany, 111, 173-181.

Wang R, Ai B, Gao BQ, Yu S, Li YY, Chen XY (2009) Spatial genetic structure and restricted gene flow in a functionally dioecious fig, Ficus pumila L. var. pumila (Moraceae). Population Ecology, 51, 307-315.

Wang YG (2017) Natural hybridization and speciation. Biodiversity Science, 25, 565-576. (in Chinese with English abstract) [王玉国 (2017) 自然杂交与物种形成. 生物多 样性, 25, 565-576.]

Ware AB, Compton SG (1992) Breakdown of pollinator specificity in an African fig tree. Biotropica, 24, 544-549.

Wei ZD (2010) On Going Co-divergence of Auriculata Complex and Its Pollinating Wasps (Ceratosolen emarginatus). PhD dissertation, Xishuangbanna Tropical Botanical Garden, Chinese Academy of Sciences, Mengla, Yunnan. (in Chinese with English abstract) [魏作东 (2010) 木瓜榕物种复合体 (Auriculata complex)和它们的传粉小蜂(Ceratosolen emarginatus)间的共同分化. 博士学位论文, 中国科学院西 双版纳热带植物园, 云南预腊.]

Wei ZD, Kobmoo N, Cruaud A, Kjellberg F (2014) Genetic structure and hybridization in the species group of Ficus auriculata: Can closely related sympatric Ficus species retain their genetic identity while sharing pollinators? Molecular Ecology, 23, 3538-3550.
Weiblen GD (2004) Correlated evolution in fig pollination. Systematic Biology, 53, 128-139.

Wendel JF, Doyle JJ (1998) Phylogenetic incongruence: Window into genome history and molecular evolution. In: Molecular Systematics of Plants II. DNA Sequencing (eds Soltis DE, Soltis PS, Doyle JJ), pp. 265-296. Kluwer Academic Publishers, Boston/Dordrecht/London.

Wiebes JT (1963) Taxonomy and host preferences of Indo-Australian fig wasps of the genus Ceratosolen (Agaonidae). Tijdeschrift Voor Entomologie, 106, 1-112.

Xu ZF (1994) Ficus-A keystone plant group in the tropical rain forests ecosystem of south Yunnan. Chinese Biodiversity, 2, 21-23. (in Chinese) [许再富 (1994) 榕树一一滇 南热带雨林生态系统中的一类关键植物. 生物多样性, 2 , 21-23.]

Yakushiji H, Morita T, Jikumaru S, Ikegami H, Azuma A, Koshita Y (2012) Interspecific hybridization of fig (Ficus carica L.) and Ficus erecta Thunb., a source of Ceratocystis canker resistance. Euphytica, 183, 39-47.

Yang LY, Machado CA, Dang XD, Peng YQ, Yang DR, Zhang DY, Liao WJ (2015) The incidence and pattern of copollinator diversification in dioecious and monoecious figs. Evolution, 69, 294-304.

Yang P (2010) Research on Mutualism of Three Ficus Species in Subsection Neomorphe and Their Pollinators. PhD dissertation, Xishuangbanna Tropical Botanical Garden, Chinese Academy of Sciences, Mengla, Yunnan. (in Chinese with English abstract) [杨培 (2010) 大果榕亚组三种榕树榕蜂互惠共生关系的研究. 博士学位论文, 中国科学院西 双版纳热带植物园, 云南敌腊.]

Yang P, Li ZB, Peng YQ, Yang DR (2012) Exchange of hosts: Can agaonid fig wasps reproduce successfully in the figs of non-host Ficus? Naturwissenschaften, 99, 199-205.

Yokoyama J, Iwatsuki K (1998) A faunal survey of fig-wasps (Chalcidoidea: Hymenoptera) distributed in Japan and their associations with figs (Ficus: Moraceae). Entomological Science, 1, 37-46.

Zhang LF, Zhang Z, Wang XM, Gao HY, Tian HZ, Li HQ (2018) Molecular phylogeny of the Ficus auriculata complex (Moraceae). Phytotaxa, 362, 39-54.

Zhao JB (2013) Cheating Evolution of Eupristina sp. in Ficus altissima. PhD dissertation, Xishuangbanna Tropical Botanical Garden, Chinese Academy of Sciences, Mengla, Yunnan. (in Chinese with English abstract) [赵江波 (2013) 高 山榕传粉小蜂由互惠向欺骗演化的机制. 博士学位论文, 中国科学院西双版纳热带植物园, 云南预腊.]

(责任编委: 严岳鸿 责任编辑: 时意专) 